\title{
JACOBI POLYNOMIALS, BERNSTEIN-TYPE INEQUALITIES AND DISPERSION ESTIMATES FOR THE DISCRETE LAGUERRE OPERATOR
}

\author{
TOM KOORNWINDER, ALEKSEY KOSTENKO, AND GERALD TESCHL
}

\begin{abstract}
The present paper is about Bernstein-type estimates for Jacobi polynomials and their applications to various branches in mathematics. This is an old topic but we want to add a new wrinkle by establishing some intriguing connections with dispersive estimates for a certain class of Schrödinger equations whose Hamiltonian is given by the generalized Laguerre operator. More precisely, we show that dispersive estimates for the Schrödinger equation associated with the generalized Laguerre operator are connected with Bernsteintype inequalities for Jacobi polynomials. We use known uniform estimates for Jacobi polynomials to establish some new dispersive estimates. In turn, the optimal dispersive decay estimates lead to new Bernstein-type inequalities.
\end{abstract}

\section{INTRODUCTION}

To set the stage, for $\alpha, \beta>-1$, let $w^{(\alpha, \beta)}(x)=(1-x)^{\alpha}(1+x)^{\beta}$ for $x \in(-1,1)$ be a Jacobi weight. The corresponding orthogonal polynomials $P_{n}^{(\alpha, \beta)}$, normalized by

$$
P_{n}^{(\alpha, \beta)}(1)=\left(\begin{array}{c}
n+\alpha \\
n
\end{array}\right)=\frac{(\alpha+1)_{n}}{n !}
$$

for all $n \in \mathbb{N}_{0}$ (see (1.21) for notation of Pochhammer symbols and binomial coefficients), are called the Jacobi polynomials. They are expressed as (terminating) Gauss hypergeometric series (1.22) by [44, (4.21.2)]

$$
\frac{P_{n}^{(\alpha, \beta)}(x)}{P_{n}^{(\alpha, \beta)}(1)}={ }_{2} F_{1}\left(\begin{array}{c}
-n, n+\alpha+\beta+1 \\
\alpha+1
\end{array} ; \frac{1-x}{2}\right) .
$$

They also satisfy Rodrigues' formula [44, (4.3.1), (4.3.2)]

$$
\begin{aligned}
P_{n}^{(\alpha, \beta)}(x) & =\sum_{k=0}^{n}\left(\begin{array}{c}
n+\alpha \\
n-k
\end{array}\right)\left(\begin{array}{c}
n+\beta \\
k
\end{array}\right)\left(\frac{x-1}{2}\right)^{k}\left(\frac{x+1}{2}\right)^{n-k} \\
& =\frac{(-1)^{n}}{2^{n} n !}(1-x)^{-\alpha}(1+x)^{-\beta} \frac{d^{n}}{d x^{n}}\left\{(1-x)^{\alpha+n}(1+x)^{\beta+n}\right\} .
\end{aligned}
$$

Note that, by (1.3), $P_{n}^{(\alpha, \beta)}(x)$ is for given $n$ a polynomial in $x, \alpha$ and $\beta$. Thus, if we don't need the orthogonality relations of the Jacobi polynomials, then we are not restricted by the bounds $\alpha, \beta>-1$.

2010 Mathematics Subject Classification. Primary 33C45, 47B36 Secondary 81U30 81Q05 Key words and phrases. Schrödinger equation, dispersive estimates, Jacobi polynomials. Research supported by the Austrian Science Fund (FWF) under Grant No. P26060. Adv. Math. 333, 796-821 (2018). 
The (squared normalized) $L^{2}$ norm of $P_{n}^{(\alpha, \beta)}$ is given by [44, (4.3.3)]

$$
\begin{aligned}
\frac{\Gamma(\alpha+\beta+2)}{2^{\alpha+\beta+1} \Gamma(\alpha+1) \Gamma(\beta+1)} \int_{-1}^{1}\left|P_{n}^{(\alpha, \beta)}(x)\right|^{2} w^{(\alpha, \beta)}(x) d x & \\
= & \frac{n+\alpha+\beta+1}{2 n+\alpha+\beta+1} \frac{(\alpha+1)_{n}(\beta+1)_{n}}{(\alpha+\beta+2)_{n} n !} .
\end{aligned}
$$

Jacobi polynomials include the ultraspherical (Gegenbauer) polynomials [44, (4.37.1)]

$$
P_{n}^{(\lambda)}(x):=\frac{(2 \lambda)_{n}}{(\lambda+1 / 2)_{n}} P_{n}^{\left(\lambda-\frac{1}{2}, \lambda-\frac{1}{2}\right)}(x),
$$

where $\lambda>-1 / 2$ (for $\lambda=0$, one needs to replace (1.6) by a suitable limit, see [44, Eq. (4.7.8)]), and the Legendre polynomials

$$
P_{n}(x):=P_{n}^{(1 / 2)}(x)=P_{n}^{(0,0)}(x)=\frac{1}{2^{n} n !} \frac{d^{n}}{d x^{n}}\left(x^{2}-1\right)^{n} .
$$

We shall denote the corresponding orthonormal polynomials by $\mathbf{p}_{n}^{(\alpha, \beta)}$ for Jacobi, $\mathbf{p}_{n}^{(\lambda)}$ for Gegenbauer, and $\mathbf{p}_{n}$ for Legendre polynomials.

The Rodrigues formula (1.4) immediately implies

$$
P_{n}^{(\alpha, \beta)}(-x)=(-1)^{n} P_{n}^{(\beta, \alpha)}(x),
$$

and hence

$$
P_{n}^{(\alpha, \beta)}(-1)=(-1)^{n}\left(\begin{array}{c}
n+\beta \\
n
\end{array}\right)=(-1)^{n} \frac{(\beta+1)_{n}}{n !} .
$$

It is well known that the absolute value of $P_{n}^{(\alpha, \beta)}$ attains its maximum at the endpoints of the interval $[-1,1]$

$$
\max _{x \in[-1,1]}\left|P_{n}^{(\alpha, \beta)}(x)\right|=\max _{x \in\{-1,1\}}\left|P_{n}^{(\alpha, \beta)}(x)\right|=\left(\begin{array}{c}
n+\max (\alpha, \beta) \\
n
\end{array}\right),
$$

if $\max (\alpha, \beta) \geq-1 / 2$ (see [44, Theorem 7.32.1]).

The asymptotic behavior of Jacobi polynomials for large $n$ is rather well understood (see, e.g., [44, Chapter VIII]), however, almost all these formulas are not uniform in $\alpha$ and $\beta$. The main focus of the present paper is on uniform estimates for

$$
(1-x)^{a}(1+x)^{b}\left|P_{n}^{(\alpha, \beta)}(x)\right|
$$

on the whole segment of orthogonality $[-1,1]$ with some $a \geq 0$ and $b \geq 0$ (which might depend on $\alpha$ and $\beta$ ). Historically, the first result of this type is Bernstein's inequality $\sqrt{1}$ for the Legendre polynomials ([44, Theorem 7.3.3])

$$
\left(1-x^{2}\right)^{1 / 4}\left|P_{n}(x)\right| \leq \frac{2}{\sqrt{\pi(2 n+1)}}, \quad x \in[-1,1],
$$

(the refined version (1.12) was proved in [4, see also [38). The constant $\sqrt{2 / \pi}$ in (1.12) is sharp. Moreover (see [44, Theorem 12.1.6]), the following expression

$$
\left(1-x^{2}\right)^{1 / 4} \sqrt{w^{(\alpha, \beta)}(x)} \mathbf{p}_{n}^{(\alpha, \beta)}(x)
$$

\footnotetext{
${ }^{1}$ In order to avoid confusions with the Bernstein inequality for (algebraic) polynomials in the unit disk $\left(\max _{|z| \leq 1}\left|P^{\prime}(z)\right| \leq n \cdot \max _{|z| \leq 1}|P(z)|\right.$, where $n$ is the degree of $P$ ), throughout the text "Bernstein's inequality" should read as "Bernstein's inequality for Legendre/Gegenbauer/Jacobi polynomials" meaning the uniform (weighted) estimate for the corresponding family of orthogonal polynomials.
} 
asymptotically equioscillates between $-\sqrt{2 / \pi}$ and $\sqrt{2 / \pi}$ when $n$ tends to infinity (the latter holds for a wider class of orthonormal polynomials) and hence a lot of effort has been put in proving the estimates for (1.11) with $a=\frac{\alpha}{2}+\frac{1}{4}$ and $b=\frac{\beta}{2}+\frac{1}{4}$. Thus, for ultraspherical polynomials the corresponding estimates can be found in [44, Theorem 7.33.2] (the case $\lambda \in(0,1)$, see also [39] for a refinement), 37] (the case $\lambda>0$ ) and [14] (the case $\lambda \geq 1$ ). In the nonsymmetric case, let us mention [8], 13 and the recent papers [20, 31, 32. Let us also mention that it was conjectured by Erdélyi, Magnus and Nevai 13 that

$$
\max _{x \in(-1,1)}\left(1-x^{2}\right)^{1 / 4} \sqrt{w^{(\alpha, \beta)}(x)}\left|\mathbf{p}_{n}^{(\alpha, \beta)}(x)\right| \leq C \max \left(1,(|\alpha|+|\beta|)^{1 / 4}\right)
$$

for all $n \in \mathbb{N}_{0}$ and $\alpha, \beta \geq-1 / 2$. Notice that a weaker bound $\mathcal{O}\left(\max \left(1,\left(\alpha^{2}+\beta^{2}\right)^{1 / 4}\right)\right)$ was proved in [13, Theorem 1]. On the other hand, the Erdélyi-Magnus-Nevai conjecture (1.14) was confirmed for all $n \in \mathbb{N}_{0}$ and $\alpha, \beta \in(-1 / 2,1 / 2)$ in [8] (with a sharp estimate of the error term, see also [15]) and for all $n \geq 6$ and $\alpha$, $\beta \geq(1+\sqrt{2}) / 4$ in [31], [32] (see also [20]).

The estimates for (1.11) with $a \neq \frac{\alpha}{2}+\frac{1}{4}$ and $b \neq \frac{\beta}{2}+\frac{1}{4}$ are much less studied, however, they are important in many applications. Let us mention only a few of them. First of all, ultraspherical polynomials arise in quantum mechanics as spherical harmonics. More precisely, the $L^{2}$ normalized spherical harmonics, which are eigenfunctions of the Laplace-Beltrami operator on the sphere $\mathbb{S}^{2}$, are given by (cf. [44, (4.7.35)])

$$
Y_{l}^{m}(\theta, \varphi):=\frac{(-1)^{m}}{\sqrt{2 \pi}} \mathrm{e}^{\mathrm{i} m \varphi} \sin ^{m}(\theta) \mathbf{p}_{l-m}^{(m+1 / 2)}(\cos (\theta))
$$

if $m \in\{0, \ldots, l\}$. Therefore, (1.11) provides uniform weighted $L^{\infty}$ estimates on eigenfunctions of the Laplace-Beltrami operator on $\mathbb{S}^{2}$. In particular, the following inequality was established in [6, Theorem 1]:

$$
\max _{x \in(-1,1)}|x|^{1 / 6}\left(1-x^{2}\right)^{m / 2+1 / 6}\left|\mathbf{p}_{n}^{(m+1 / 2)}(x)\right| \leq C(n+m+1)^{1 / 6},
$$

with some $C>0$, which does not depend on $n, m \in \mathbb{N}_{0}$. Moreover, (1.16) and Krasikov's estimates 32 were employed in [6] and [42, respectively, in order to obtain bounds on the number of samples necessary for recovering sparse eigenfunction expansions on surfaces of revolution.

The next example is also widely known. More precisely, Jacobi polynomials appear as coefficients of the so-called Wigner $d$-matrix (see Theorem 4.2). Thus Bernstein-type estimates imply uniform bounds on a complete set of matrix coefficients for irreducible representations of $\mathrm{SU}(2)$ (see [20 and Section 4 below). Furthermore, these inequalities play a very important role in the study of simple Lie groups. Namely, the Bernstein inequality and the Haagerup-Schlichtkrull inequality (see (4.10) below) were used in [36] and [18, [19], respectively, to study the approximation property of Haagerup and Kraus [17 for connected simple Lie groups. 
Finally, our interest in the estimates of the type (1.11) comes from the so-called dispersive estimates for discrete Laguerre operators

$$
H_{\alpha}:=\left(\begin{array}{cccc}
1+\alpha & \sqrt{1+\alpha} & 0 & \ldots \\
\sqrt{1+\alpha} & 3+\alpha & \sqrt{2(2+\alpha)} & \ldots \\
0 & \sqrt{2(2+\alpha)} & 5+\alpha & \ldots \\
\vdots & \vdots & \vdots & \ddots
\end{array}\right), \quad \alpha>-1,
$$

acting in $\ell^{2}\left(\mathbb{N}_{0}\right)$. Explicitly, $H_{\alpha}=\left(h_{n, m}^{(\alpha)}\right)_{n, m \in \mathbb{N}_{0}}$ with $h_{n, m}^{(\alpha)}=0$ if $|n-m|>1$ and

$$
h_{n, n}^{(\alpha)}=2 n+1+\alpha, \quad h_{n, n+1}^{(\alpha)}=h_{n+1, n}^{(\alpha)}=\sqrt{(n+1)(n+1+\alpha)}, \quad n \in \mathbb{N}_{0} .
$$

It is a special case of a self-adjoint Jacobi operator whose generalized eigenfunctions are precisely the Laguerre polynomials $L_{n}^{(\alpha)}$, explaining our name for (1.17).

The operator $H_{\alpha}$ features prominently in the recent study of nonlinear waves in $(2+1)$-dimensional noncommutative scalar field theory [1, 2, 16. The coefficient $\alpha$ in (1.17) can be seen as a measure of the delocalization of the field configuration and it is related to the planar angular momentum [2]. In particular, $\alpha=0$ corresponds to spherically symmetric waves and it has attracted further interest in [7, 33, 34, 35. As this operator appears as the linear part in the nonlinear Klein-Gordon equation [7] and the nonlinear Schrödinger equation

$$
\mathrm{i} \dot{\psi}(t, n)=H_{0} \psi(t, n)-|\psi(t, n)|^{2 \sigma} \psi(t, n), \quad \sigma \in \mathbb{N}, \quad(t, n) \in \mathbb{R}_{+} \times \mathbb{N}_{0},
$$

investigated in the recent work of Krueger and Soffer [33, 34, 35], dispersive estimates play a crucial role in the understanding of stability of the soliton manifolds appearing in these models (for further details see [7, 16, 33, 34, 35). It turns out that the required dispersive decay estimates for the evolution group $\mathrm{e}^{-\mathrm{i} t H_{\alpha}}$ lead to Bernstein-type estimates for (1.11) (see 28 and Sections 647 below). All these connections are mathematically very appealing and we hope that this note will stimulate further research in this direction.

In conclusion let us briefly outline the content of the paper. In the next section we introduce discrete Laguerre operators and briefly review their spectral properties. In Section 3, we present a connection between discrete Laguerre operators $H_{\alpha}$ and Jacobi polynomials. More precisely, we show that the kernel of the evolution group $\mathrm{e}^{\mathrm{i} t H_{\alpha}}$ can be expressed by means of Jacobi polynomials (Theorem 3.1). This result establishes a connection between uniform estimates for (1.11) and dispersive estimates for the evolution group $\mathrm{e}^{\mathrm{i} t H_{\alpha}}$. In Section 4, we review the connection between irreducible representations of $\mathrm{SU}(2)$ and Jacobi polynomials. The latter, in particular, implies the estimates for (1.11) with $a=\frac{\alpha}{2}$ and $b=\frac{\beta}{2}$ when $\alpha, \beta \in \mathbb{N}_{0}$ (see (4.8) and (4.9)). In Section 5 we prove the following Bernstein-type estimate

$$
\left(\frac{1+x}{2}\right)^{\beta / 2}\left|P_{n}^{(\alpha, \beta)}(x)\right| \leq\left(\begin{array}{c}
n+\alpha \\
n
\end{array}\right), \quad x \in[-1,1],
$$

if $\beta \geq 0$ and $\alpha \geq \beta-\lfloor\beta\rfloor$.

Finally, Bernstein-type inequalities enable us to prove the decay estimates for the evolution group $\mathrm{e}^{-\mathrm{i} t H_{\alpha}}$, which we discuss in Section 6. First of all, using the known Bernstein-type inequalities, we prove the decay estimates of order $\mathcal{O}\left(t^{-1}\right)$ (Theorem 6.1) and $\mathcal{O}\left(t^{-1 / 2}\right)$ if $\alpha \geq 0$, however, with a better behavior of weights $\sigma$ 
(Theorem 6.3). On the other hand, the new inequality (1.19) enables us to show that

$$
\left\|\mathrm{e}^{-\mathrm{i} t H_{\alpha}}\right\|_{\ell^{1}\left(\sigma_{\alpha}\right) \rightarrow \ell^{\infty}\left(\sigma_{\alpha}^{-1}\right)}=\left(\frac{1}{1+t^{2}}\right)^{\frac{1+\alpha}{2}}, \quad t \in \mathbb{R},
$$

for all $\alpha \geq 0$, with the weights $\sigma_{\alpha}$ given by $\sigma_{\alpha}=\left\{\left(\begin{array}{c}n+\alpha \\ n\end{array}\right)^{1 / 2}\right\}_{n \geq 0}$ (see Theorem 6.5). We finish our paper with some further comments on new Bernstein-type inequalities and certain parallels between dispersive estimates for discrete Laguerre operators and one-dimensional spherical Schrödinger operators (see Section 7 ).

Notation. $\mathbb{R}$ and $\mathbb{C}$ have the usual meaning. Also write $\mathbb{R}_{+}:=(0, \infty), \mathbb{N}:=$ $\{1,2, \ldots\}$ and $\mathbb{N}_{0}:=\mathbb{N} \cup\{0\}$. By $\Gamma$ is denoted the classical gamma function [40, $(5.2 .1)$. For $x \in \mathbb{C}$ and $n \in \mathbb{N}_{0}$

$$
(x)_{n}:=\left\{\begin{array}{ll}
x(x+1) \cdots(x+n-1), & n \in \mathbb{N} \\
1, & n=0
\end{array} ; \quad\left(\begin{array}{c}
n+x \\
n
\end{array}\right):=\frac{(x+1)_{n}}{n !}\right.
$$

denote the Pochhammer symbol [40, (5.2.4)] and the binomial coefficient, respectively. Notice that for $-x \notin \mathbb{N}_{0}$

$$
(x)_{n}=\frac{\Gamma(x+n)}{\Gamma(x)}, \quad\left(\begin{array}{c}
n+x \\
n
\end{array}\right)=\frac{\Gamma(x+n+1)}{\Gamma(x+1) \Gamma(n+1)} .
$$

Moreover, the above formulas allow to define the Pochhammer symbol and the binomial coefficient for noninteger $x, n>0$. Finally, for $-c \notin \mathbb{N}_{0}$ the Gauss hypergeometric function [40, (15.2.1)] is defined by

$$
{ }_{2} F_{1}\left(\begin{array}{c}
a, b \\
c
\end{array} ; z\right):=\sum_{k=0}^{\infty} \frac{(a)_{k}(b)_{k}}{(c)_{k} k !} z^{k} \quad\left(|z|<1 \text { or else }-a \text { or }-b \in \mathbb{N}_{0}\right) .
$$

\section{Spectral properties of the discrete Laguerre operators}

We start with a precise definition of the operator $H_{\alpha}$ associated with the Jacobi matrix (1.17). For a sequence $u=\left\{u_{n}\right\}_{n \geq 0}$ we define the difference expression $\tau_{\alpha}: u \mapsto \tau_{\alpha} u$ by setting

$$
\left(\tau_{\alpha} u\right)_{n}:=\sqrt{n(n+\alpha)} u_{n-1}+(2 n+1+\alpha) u_{n}+\sqrt{(n+1)(n+1+\alpha)} u_{n+1},
$$

where $u_{-1}:=0$ for notational convenience. Then the operator $H_{\alpha}$ associated with the Jacobi matrix (1.17) is defined by

$$
\begin{aligned}
H_{\alpha}: \mathcal{D}_{\max } & \rightarrow \ell^{2}\left(\mathbb{N}_{0}\right), \\
u & \mapsto \tau_{\alpha} u
\end{aligned}
$$

where $\mathcal{D}_{\max }=\left\{u \in \ell^{2}\left(\mathbb{N}_{0}\right) \mid \tau_{\alpha} u \in \ell^{2}\left(\mathbb{N}_{0}\right)\right\}$. The spectral properties of $H_{\alpha}$ are well known. For the sake of completeness we collect them in the following theorem and give a short proof.

Theorem 2.1. Let $\alpha>-1$. Then:

(i) The operator $H_{\alpha}$ is a positive self-adjoint operator.

(ii) The spectrum of $H_{\alpha}$ is purely absolutely continuous and coincides with $[0, \infty)$. 
(iii) The Weyl function and the corresponding spectral measure are given by

$$
\begin{aligned}
m_{\alpha}(z) & =\frac{1}{\Gamma(\alpha+1)} \int_{0}^{+\infty} \frac{\mathrm{e}^{-\lambda} \lambda^{\alpha}}{\lambda-z} d \lambda=\mathrm{e}^{-z} E_{1+\alpha}(-z), \quad z \in \mathbb{C} \backslash[0, \infty), \\
d \rho_{\alpha}(\lambda) & =\frac{\mathbb{1}_{\mathbb{R}_{+}}(\lambda)}{\Gamma(\alpha+1)} \mathrm{e}^{-\lambda} \lambda^{\alpha} d \lambda, \quad \lambda \in \mathbb{R},
\end{aligned}
$$

where $E_{p}(z):=z^{p-1} \int_{z}^{\infty} \mathrm{e}^{-t} t^{-p} d t$ denotes the principal value of the generalized exponential integral [40, (8.19.2)].

Proof. (i) Self-adjointness clearly follows from the Carleman test (see, e.g., [3], 45, (2.165)]). Nonnegativity as well as item (ii) immediately follow from (iii), so let us prove (iii). Notice that the orthogonal polynomials for $H_{\alpha}$ are given by

$$
P_{\alpha, n}(z)=\frac{(-1)^{n}}{\sigma_{\alpha}(n)} L_{n}^{(\alpha)}(z), \quad n \in \mathbb{N}_{0}
$$

where

$$
\sigma_{\alpha}(n)=\sqrt{L_{n}^{(\alpha)}(1)}=\left(\begin{array}{c}
n+\alpha \\
n
\end{array}\right)^{1 / 2}
$$

and $L_{n}^{(\alpha)}$ are the Laguerre polynomials [44, Section 5.1]

$$
\frac{L_{n}^{(\alpha)}(z)}{L_{n}^{(\alpha)}(1)}={ }_{1} F_{1}\left(\begin{array}{c}
-n \\
\alpha+1
\end{array} ; z\right)=\sum_{k=0}^{n} \frac{(-n)_{k}}{(\alpha+1)_{k} k !} z^{k} .
$$

The recurrence formula for the Laguerre polynomials [44, (5.1.10)] implies that $u:=$ $\left\{P_{\alpha, n}(z)\right\}_{n \in \mathbb{N}_{0}}$ satisfies $\left(\tau_{\alpha} u\right)_{n}=z u_{n}$ for all $n \geq 0$. Furthermore, the polynomials $L_{n}^{(\alpha)}$ satisfy the orthogonality relations [44, (5.1.1)]

$$
\frac{1}{\Gamma(\alpha+1)} \int_{0}^{\infty} L_{n}^{(\alpha)}(\lambda) L_{k}^{(\alpha)}(\lambda) \mathrm{e}^{-\lambda} \lambda^{\alpha} d \lambda=\left(\begin{array}{c}
n+\alpha \\
n
\end{array}\right) \delta_{n k}, \quad n, k \in \mathbb{N}_{0} .
$$

Therefore, (2.7) and (i) imply that $d \rho_{\alpha}$ is the spectral measure of $H_{\alpha}$, that is, $H_{\alpha}$ is unitarily equivalent to a multiplication operator in $L^{2}\left(\mathbb{R}_{+}, d \rho_{\alpha}\right)$. It remains to note that the corresponding Weyl function is the Stieltjes transform of the measure $d \rho_{\alpha}$ (cf. e.g. [45, Chapter 2]).

Remark 2.2. The operator $H_{\alpha}$, when restricted to $\ell_{c}^{2}\left(\mathbb{N}_{0}\right)$, can be seen as occurring in a discrete series representation of the Lie algebra $\mathfrak{s l}(2, \mathbb{R})$. First define operators $A, X, Y$ on this linear span by

$A u_{n}:=(2 n+\alpha+1) u_{n}, X u_{n}:=\sqrt{(n+1)(n+\alpha+1)} u_{n+1}, Y u_{n}:=\sqrt{n(n+\alpha)} u_{n-1}$.

They satisfy the commutator relations

$$
[A, X]=2 X, \quad[A, Y]=-2 Y, \quad[X, Y]=-A .
$$

Now consider the skew-hermitian operators

$$
J_{0}:=X-Y, \quad J_{+}:=\frac{1}{2} \mathrm{i}(-A+X+Y), \quad J_{-}:=\frac{1}{2} \mathrm{i}(A+X+Y)=\frac{1}{2} \mathrm{i} H_{\alpha} .
$$

They form an $\mathfrak{s l}(2, \mathbb{R})$ triple:

$$
\left[J_{0}, J_{+}\right]=2 J_{+}, \quad\left[J_{0}, J_{-}\right]=-2 J_{-}, \quad\left[J_{+}, J_{-}\right]=J_{0} .
$$

Thus we have a representation of the Lie algebra $\mathfrak{s l}(2, \mathbb{R})$ :

$$
\left(\begin{array}{cc}
1 & 0 \\
0 & -1
\end{array}\right) \rightarrow J_{0}, \quad\left(\begin{array}{ll}
0 & 1 \\
0 & 0
\end{array}\right) \rightarrow J_{+}, \quad\left(\begin{array}{ll}
0 & 0 \\
1 & 0
\end{array}\right) \rightarrow J_{-} .
$$


In particular, $\left(\begin{array}{cc}0 & -1 \\ 1 & 0\end{array}\right)$, which spans the Lie subalgebra of the subgroup $K:=S O$ (2) of $\mathfrak{s l}(2, \mathbb{R})$, is mapped in this representation to iA. If we compare with [25, Section 7], which builds on [5. Section 3], we see that this representation, when exponentiated to a unitary representation of the Lie group $\mathrm{SL}(2, \mathbb{R})$, is a so-called discrete series representation $D_{\frac{1}{2}(\alpha+1)}^{+}$of $\mathrm{SL}(2, \mathbb{R})$ for $\alpha \in \mathbb{N}_{0}$, and otherwise, for real $\alpha>-\frac{1}{2}$, a similar representation of the universal covering group of $\mathrm{SL}(2, \mathbb{R})$ (see [4]).

\section{The evolution group $\mathrm{e}^{-\mathrm{i} t H_{\alpha}}$}

In this and the following sections we look at the one-dimensional discrete Schrödinger equation

$$
\mathrm{i} \dot{\psi}(t, n)=H_{\alpha} \psi(t, n), \quad(t, x) \in \mathbb{R} \times \mathbb{N}_{0},
$$

associated with the Laguerre operator $H_{\alpha}$ defined in the previous section. We begin by establishing a connection between the discrete Laguerre operators and Jacobi polynomials, which follows from the fact that the Laplace transform of a product of two Laguerre polynomials is expressed by means of a terminating Gauss hypergeometric series.

Theorem 3.1. Let $\alpha>-1$. The kernell of the operator $\mathrm{e}^{-\mathrm{i} t H_{\alpha}}$ is given by

$$
\begin{aligned}
& \mathrm{e}^{-\mathrm{i} t H_{\alpha}}(n, m)=\mathrm{e}^{-\mathrm{i} t H_{\alpha}}(m, n) \\
& =\frac{1}{(1+\mathrm{i} t)^{1+\alpha}}\left(\frac{t+\mathrm{i}}{t-\mathrm{i}}\right)^{n}\left(\frac{t}{t-\mathrm{i}}\right)^{m-n} \frac{\sigma_{\alpha}(m)}{\sigma_{\alpha}(n)} P_{n}^{(\alpha, m-n)}\left(\frac{t^{2}-1}{t^{2}+1}\right)
\end{aligned}
$$

for all $n, m \in \mathbb{N}_{0}$.

Proof. Similar to the case $\alpha=0$ (see [28]), one gets by employing Stone's formula (cf., e.g. [46, §4.1])

$$
\mathrm{e}^{-\mathrm{i} t H_{\alpha}}(n, m)=\frac{(-1)^{n+m}}{\sigma_{\alpha}(n) \sigma_{\alpha}(m) \Gamma(\alpha+1)} \int_{0}^{\infty} \mathrm{e}^{-\mathrm{i} t \lambda} L_{n}^{(\alpha)}(\lambda) L_{m}^{(\alpha)}(\lambda) \mathrm{e}^{-\lambda} \lambda^{\alpha} d \lambda
$$

for all $n, m \in \mathbb{N}_{0}$. It follows from (3.3) that every element of the kernel of the operator $\mathrm{e}^{-\mathrm{i} t H_{\alpha}}$ is the Laplace transform of a product of two Laguerre polynomials. Then using [12, (4.11.35)] and [40, (15.8.7)] together with Euler's transformation [40, (15.8.1)], after lengthy but straightforward calculations one arrives at (3.2).

Remark 3.2. It is interesting to mention that the unitarity of $\mathrm{e}^{-\mathrm{i} t H_{\alpha}}$ is equivalent to the orthogonality relations for the Meixner polynomials [40, (18.20.7)]

$$
M_{n}(x ; \beta, c):={ }_{2} F_{1}\left(\begin{array}{c}
-n,-x \\
\beta
\end{array} ;-c^{-1}\right) .
$$

Namely,

$$
M_{n}(x ; \beta, c)=\frac{n !}{c^{n}(\beta)_{n}} P_{n}^{(\beta-1, x-n)}(2 c-1),
$$

and then equation (3.2) reads

$$
\frac{\mathrm{e}^{-\mathrm{i} t H_{\alpha}}(n, m)}{\sigma_{\alpha}(m) \sigma_{\alpha}(n)}=\frac{1}{(1+\mathrm{i} t)^{1+\alpha}}\left(\frac{-\mathrm{i} t}{1+\mathrm{i} t}\right)^{n+m} M_{n}\left(m ; \alpha+1, \frac{t^{2}}{1+t^{2}}\right) .
$$

\footnotetext{
${ }^{2}$ In analogy with the (integral) kernel of an integral operator we speak about the (summation) kernel of a summation operator acting by a matrix.
} 
It remains to note that the orthogonality relations are [40, Table 18.19.1] (with positive weights if $\beta>0$ and $0<c<1$ )

$$
(1-c)^{\beta} \sum_{x=0}^{\infty} \frac{(\beta)_{x} c^{x}}{x !} M_{n}(x ; \beta, c) M_{k}(x ; \beta, c)=\frac{n !}{(\beta)_{n} c^{n}} \delta_{n k} .
$$

Remark 3.3. We continue Remark 2.2 and assume, for convenience, that $\alpha \in \mathbb{N}_{0}$, so that we can refer to [25, Section 7]. In the realization of the discrete series representation given there, a K-basis [25, (7.16)] is given in terms of Laguerre polynomials and the $K-K$ matrix elements [25. (7.20)] are in terms of Meixner polynomials. This provides a further explanation of the observations in Remark 3.2.

The next result provides recurrence relations for the kernel of $\mathrm{e}^{\mathrm{i} t H_{\alpha}}$.

Corollary 3.4. Let $\alpha>-1$ and $n \leq m$. Then

$$
\begin{aligned}
\mathrm{e}^{-\mathrm{i} t H_{\alpha}}(n+1, m+1)= & \sqrt{\frac{(m+1)(m+1+\alpha)}{(n+1)(n+1+\alpha)}} \frac{\mathrm{i}+t}{\mathrm{i}-t} \mathrm{e}^{-\mathrm{i} t H_{\alpha}}(n, m) \\
& +\frac{n+m+\alpha+2}{\sqrt{(n+1)(n+1+\alpha)}} \frac{t}{\mathrm{i}-t} \mathrm{e}^{-\mathrm{i} t H_{\alpha}}(n, m+1) \\
= & \frac{n+m+\alpha+2}{\sqrt{(n+1)(m+1)} \frac{1}{1+\mathrm{i} t} \mathrm{e}^{-\mathrm{i} t H_{\alpha+1}}(n, m)} \\
& +\sqrt{\frac{(n+\alpha+1)(m+1+\alpha)}{(n+1)(m+1)}} \frac{\mathrm{i}+t}{\mathrm{i}-t} \mathrm{e}^{-\mathrm{i} t H_{\alpha}}(n, m) .
\end{aligned}
$$

Proof. Using the recurrence relations for Jacobi polynomials (see [44, (4.5.4)]):

$$
\begin{aligned}
P_{n+1}^{(\alpha, \beta)}(x) & =\frac{n+\alpha+1}{n+1} P_{n}^{(\alpha, \beta)}(x)-\frac{2 n+\alpha+\beta+2}{n+1} \frac{1-x}{2} P_{n}^{(\alpha+1, \beta)}(x) \\
& =\frac{2 n+\alpha+\beta+2}{n+1} \frac{1+x}{2} P_{n}^{(\alpha, \beta+1)}(x)-\frac{n+\beta+1}{n+1} P_{n}^{(\alpha, \beta)}(x),
\end{aligned}
$$

straightforward calculations complete the proof.

We collect some special cases explicitly for later use.

Corollary 3.5. (i) In the case $n=0$ we have

$$
\mathrm{e}^{-\mathrm{i} t H_{\alpha}}(0, m)=\frac{1}{(1+\mathrm{i} t)^{1+\alpha}}\left(\frac{-\mathrm{i} t}{1+\mathrm{i} t}\right)^{m} \sqrt{\frac{(\alpha+1)_{m}}{m !}}, \quad m \in \mathbb{N}_{0} .
$$

(ii) In the case $n=1$ we have for $m \in \mathbb{N}$

$$
\mathrm{e}^{-\mathrm{i} t H_{\alpha}}(1, m)=\frac{1}{(1+\mathrm{i} t)^{1+\alpha}}\left(\frac{-\mathrm{i} t}{1+\mathrm{i} t}\right)^{m+1} \frac{(1+\alpha) t^{2}-m}{t^{2}} \sqrt{\frac{(\alpha+2)_{m-1}}{m !}} .
$$

(iii) In the case $n=m$ we have

$$
\mathrm{e}^{-\mathrm{i} t H_{\alpha}}(m, m)=\frac{1}{(1+\mathrm{i} t)^{1+\alpha}}\left(\frac{t+\mathrm{i}}{t-\mathrm{i}}\right)^{m} P_{m}^{(\alpha, 0)}\left(\frac{t^{2}-1}{t^{2}+1}\right), \quad m \in \mathbb{N}_{0} .
$$

Proof. Just observe

$$
P_{0}^{(\alpha, m)}(z)=1, \quad P_{1}^{(\alpha, m-1)}(z)=-m+(m+1+\alpha) \frac{z+1}{2} .
$$


Let us also mention the following estimate.

Lemma 3.6. If $\alpha>-1$ and $\beta+n \in \mathbb{N}_{0}$, then

$$
\left(\frac{1-x}{2}\right)^{(\alpha+1) / 2}\left(\frac{1+x}{2}\right)^{\beta / 2}\left|P_{n}^{(\alpha, \beta)}(x)\right| \leq\left(\frac{\Gamma(n+\alpha+1) \Gamma(n+\beta+1)}{\Gamma(n+1) \Gamma(n+\alpha+\beta+1)}\right)^{1 / 2}
$$

for all $x \in[-1,1]$ and $n \in \mathbb{N}_{0}$.

Proof. Noting that $\left|\mathrm{e}^{-\mathrm{i} t H_{\alpha}}(n, m)\right| \leq 1$ for all $t \in \mathbb{R}$ since $\mathrm{e}^{-\mathrm{i} t H_{\alpha}}$ is a unitary group on $\ell^{2}$, after the change of variables

$$
x=x(t):=\frac{t^{2}-1}{t^{2}+1}, \quad t \in[0, \infty),
$$

in (3.2), we arrive at (3.13).

Remark 3.7. The estimate (3.13) is of course weaker than (4.8) (see below), however, it holds for a larger range of parameters. Furthermore note that Lemma 3.6 is also a consequence of (3.7) and (3.5).

It is not difficult to see that the weighted $\ell^{1} \rightarrow \ell^{\infty}$ estimates for the evolution group $\mathrm{e}^{-\mathrm{it} H_{\alpha}}$ are closely connected with Bernstein-type estimates for Jacobi polynomials. Indeed, taking absolute values in (3.2) we get

$$
\left|\mathrm{e}^{-\mathrm{i} t H_{\alpha}}(n, m)\right|=\frac{\sigma_{\alpha}(m)}{\sigma_{\alpha}(n)}\left(\frac{1}{1+t^{2}}\right)^{\frac{1+\alpha}{2}}\left(\frac{t^{2}}{1+t^{2}}\right)^{\frac{m-n}{2}}\left|P_{n}^{(\alpha, m-n)}\left(\frac{t^{2}-1}{t^{2}+1}\right)\right|,
$$

for all $t \in \mathbb{R}$. With the rough inequality $t^{2} /\left(1+t^{2}\right)<1$ one immediately obtains the following estimates.

Lemma 3.8. Let $\alpha>-1$. Then

$$
\left(1+t^{2}\right)^{\frac{1+\alpha}{2}}\left|\mathrm{e}^{-\mathrm{i} t H_{\alpha}}(n, m)\right| \leq \begin{cases}\sigma_{\alpha}(n) \sigma_{\alpha}(m), & \alpha \geq|m-n|, \\
\frac{\sigma_{\alpha}(m)}{\sigma_{\alpha}(n)}\left(\begin{array}{c}
m \\
n
\end{array}\right), & m-n \geq \alpha, \\
\frac{\sigma_{\alpha}(n)}{\sigma_{\alpha}(m)}\left(\begin{array}{c}
n \\
m
\end{array}\right), & n-m \geq \alpha .\end{cases}
$$

for all $t \in \mathbb{R}$, and

$$
\lim _{t \rightarrow+\infty}\left(1+t^{2}\right)^{\frac{1+\alpha}{2}}\left|\mathrm{e}^{-\mathrm{i} t H_{\alpha}}(n, m)\right|=\sigma_{\alpha}(n) \sigma_{\alpha}(m)
$$

for every fixed $n, m \in \mathbb{N}_{0}$.

Proof. The standard estimate (1.10) applied to (3.15) gives (3.16). Moreover, (3.15) together with (1.9) implies (3.17).

Lemma 3.8 indicates a decay of order $O\left(|t|^{-(1+\alpha)}\right)$ for $\mathrm{e}^{-\mathrm{i} t H_{\alpha}}(n, m)$ if one uses weighted spaces. In fact, we shall show in Section 6 that for $\alpha \geq 0$ the optimal weights for this decay are given by (2.5). Let us only record the following special cases which can be established directly from Corollary 3.5

Corollary 3.9. Suppose $\alpha \geq 0$.

(i) In the case $n=0$ we have for all $m \in \mathbb{N}_{0}$

$$
\left(1+t^{2}\right)^{\frac{1+\alpha}{2}}\left|\mathrm{e}^{-\mathrm{i} t H_{\alpha}}(0, m)\right| \leq \sigma_{\alpha}(m), \quad t \in \mathbb{R} .
$$


(ii) In the case $n=1$ we have for all $m \in \mathbb{N}_{0}$

$$
\left(1+t^{2}\right)^{\frac{1+\alpha}{2}}\left|\mathrm{e}^{-\mathrm{i} t H_{\alpha}}(1, m)\right| \leq \sigma_{\alpha}(1) \sigma_{\alpha}(m), \quad t \in \mathbb{R} .
$$

(iii) In the case $n=m \in \mathbb{N}_{0}$ we have

$$
\left(1+t^{2}\right)^{\frac{1+\alpha}{2}}\left|\mathrm{e}^{-\mathrm{i} t H_{\alpha}}(m, m)\right| \leq \sigma_{\alpha}(m)^{2}, \quad t \in \mathbb{R} .
$$

Proof. (i) and (iii) are immediate from Corollary 3.5. This works for (ii) as well if $\alpha \geq|m-1|$ or if $m=0$. Otherwise we use for (ii) the new variable $x=t^{2} /\left(1+t^{2}\right)$, so that (ii) is equivalent to

$$
\max _{x \in[0,1]}\left|f_{m}(x)\right| \leq 1+\alpha, \quad f_{m}(x)=x^{\frac{m-1}{2}}((m+1+\alpha) x-m)
$$

Notice that

$$
f_{1}(x)=(2+\alpha) x-1
$$

and hence

$$
\max _{x \in[0,1]}\left|f_{1}(x)\right|=\max (-1,1+\alpha)=1+\alpha .
$$

For $m>1$ one computes

$$
f_{m}^{\prime}(x)=x^{\frac{m-3}{2}}\left(\frac{m-1}{2}((m+1+\alpha) x-m)+(m+1+\alpha) x\right) .
$$

Therefore,

$$
\max \left|f_{m}(x)\right|=\max \left(\left|f_{m}(0)\right|,\left|f_{m}(1)\right|,\left|f_{m}\left(x_{0}\right)\right|\right)=\max \left(\left|f_{m}\left(x_{0}\right)\right|, 1+\alpha\right)
$$

where

Moreover,

$$
x_{0}=\frac{m(m-1)}{(m+1)(m+1+\alpha)} .
$$

$$
\begin{aligned}
\left|f_{m}\left(x_{0}\right)\right|=\frac{2 m}{m+1} & \left(\frac{m(m-1)}{(m+1)(m+1+\alpha)}\right)^{\frac{m-1}{2}} \\
& <2\left(\left(\frac{m}{m+1}\right)^{m}\left(\frac{m-1}{m}\right)^{m-1}\right)^{1 / 2} \leq \frac{2 \sqrt{2}}{3}
\end{aligned}
$$

for $m \geq 2$ since the sequence $\left\{\left(\frac{m}{m+1}\right)^{m}\right\}_{m \geq 1}$ is strictly decreasing.

We finish this section with another representation for the kernel of the evolution group. Define the following functions

$$
F_{n}^{(\alpha)}(t)=\frac{1}{(1 / 2+\mathrm{i} t)^{1+\alpha}}\left(\frac{\mathrm{i} t-1 / 2}{\mathrm{i} t+1 / 2}\right)^{n}
$$

and

$$
G_{n}^{(\alpha)}(t)=\frac{1}{1 / 2+\mathrm{i} t} \sum_{k=0}^{n}\left(\begin{array}{c}
k+\alpha-1 \\
k
\end{array}\right)\left(\frac{\mathrm{i} t-1 / 2}{\mathrm{i} t+1 / 2}\right)^{n-k},
$$

for all $n \in \mathbb{N}_{0}$ and $t \in \mathbb{R}$. Note that the right-hand side of (3.22) involves the truncated binomial series [11, Section 2.5.4]

$$
\sum_{k=0}^{n} \frac{(\alpha)_{k}}{k !} z^{k}={ }_{2} F_{1}\left(\begin{array}{c}
-n, \alpha \\
-n
\end{array} ; z\right) .
$$


Theorem 3.10. Let $F_{n}^{(\alpha)}$ and $G_{m}^{(\alpha)}$ be given by (3.21) and (3.22). Then

$$
\mathrm{e}^{-\mathrm{i} t H_{\alpha}}(n, m)=(-1)^{n+m} \frac{\sigma_{\alpha}(n)}{\sigma_{\alpha}(m)}\left(F_{n}^{(\alpha)} * G_{m}^{(\alpha)}\right)(t),
$$

where $(f * g)(t)=\frac{1}{2 \pi} \int_{\mathbb{R}} f(x) g(t-x) d x$ is the convolution of $f$ and $g$.

Proof. Notice that by [12, (4.11.28)]

$\frac{\Gamma(n+1)}{\Gamma(n+\alpha+1)} \int_{0}^{\infty} \mathrm{e}^{-\mathrm{i} t \lambda} L_{n}^{(\alpha)}(\lambda) \mathrm{e}^{-\lambda / 2} \lambda^{\alpha} d \lambda=\frac{1}{(1 / 2+\mathrm{i} t)^{1+\alpha}}\left(\frac{\mathrm{i} t-1 / 2}{\mathrm{i} t+1 / 2}\right)^{n}=F_{n}^{(\alpha)}(t)$, and by [12, (4.11.27)]

$$
\int_{0}^{\infty} \mathrm{e}^{-\mathrm{i} t \lambda} L_{m}^{(\alpha)}(\lambda) \mathrm{e}^{-\lambda / 2} d \lambda=\frac{1}{1 / 2+\mathrm{i} t} \sum_{k=0}^{m}\left(\begin{array}{c}
k+\alpha-1 \\
k
\end{array}\right)\left(\frac{\mathrm{i} t-1 / 2}{\mathrm{i} t+1 / 2}\right)^{m-k}=G_{m}^{(\alpha)}(t) .
$$

It remains to note that the Fourier transform of a product of two $L^{1}$ functions is equal to the convolution of their Fourier transforms.

\section{Irreducible Representations of SU(2) and Jacobi polynomials}

The theory of representations of Lie groups provides a unified point of view on the theory of basic classes of special functions. In particular, the connection between irreducible representations of the special unitary group $\mathrm{SU}(2)$ and Jacobi polynomials is widely known. In this section we give a brief account of this connection (for a detailed discussion we refer to [26, [47, Ch. III], 48, Ch. 6]). First, recall that a group homomorphism $\varrho: \mathcal{G} \rightarrow \mathrm{GL}(\mathcal{H})$ of a group $\mathcal{G}$ into a group of all invertible linear transformation $\mathrm{GL}(\mathcal{H})$ on a finite dimensional complex linear space $\mathcal{H}$ is called a representation of $\mathcal{G}$ (by linear operators). The dimension of $\mathcal{H}$ is called the degree of the representation $\varrho$. A linear subspace $\tilde{\mathcal{H}} \subset \mathcal{H}$ is called invariant with respect to the representation $\varrho$ of $\mathcal{G}$ if $\varrho(g) \tilde{\mathcal{H}} \subset \tilde{\mathcal{H}}$ for all $g \in \mathcal{G}$. A representation $\varrho$ is called irreducible if $\{0\}$ and $\mathcal{H}$ are the only invariant subspaces.

In order to construct an irreducible representation of $\mathrm{SU}(2)$ of degree $d \in \mathbb{N}$ one needs to consider the space $\mathcal{H}_{d}$ of homogeneous polynomials of degree $d-1$. Set $l:=(d-1) / 2$. The inner product on $\mathcal{H}_{d}$ is defined by the requirement that the normalized monomials

$$
\psi_{k}^{d}\left(z_{1}, z_{2}\right)=\left(\begin{array}{c}
2 l \\
l-k
\end{array}\right)^{1 / 2} z_{1}^{l-k} z_{2}^{l+k}, \quad k \in\{-l,-l+1, \ldots, l-1, l\},
$$

form an orthonormal basis.

The group $\mathrm{SU}(2)$ consists of all $2 \times 2$ unitary matrices of determinant 1 . It is immediate to check that each $A \in \mathrm{SU}(2)$ has the form

$$
A=\left(\begin{array}{cc}
a & b \\
-b^{*} & a^{*}
\end{array}\right), \quad|a|^{2}+|b|^{2}=1,
$$

where $z^{*}$ denotes the complex conjugate of $z$, and hence $\mathrm{SU}(2)$ is homeomorphic to the unit sphere $\mathbb{S}^{3}$ in $\mathbb{R}^{4}$. Moreover, $A$ admits the following decomposition

$$
\begin{aligned}
A= & A(\phi, \theta, \varphi)=\left(\begin{array}{cc}
\cos (\theta) \mathrm{e}^{\mathrm{i}(\phi+\varphi)} & -\sin (\theta) \mathrm{e}^{\mathrm{i}(\phi-\varphi)} \\
\sin (\theta) \mathrm{e}^{-\mathrm{i}(\phi-\varphi)} & \cos (\theta) \mathrm{e}^{-\mathrm{i}(\phi+\varphi)}
\end{array}\right) \\
& =\left(\begin{array}{cc}
\mathrm{e}^{\mathrm{i} \phi} & 0 \\
0 & \mathrm{e}^{-\mathrm{i} \phi}
\end{array}\right)\left(\begin{array}{cc}
\cos (\theta) & -\sin (\theta) \\
\sin (\theta) & \cos (\theta)
\end{array}\right)\left(\begin{array}{cc}
\mathrm{e}^{\mathrm{i} \varphi} & 0 \\
0 & \mathrm{e}^{-\mathrm{i} \varphi}
\end{array}\right)=A(\phi, 0,0) A(0, \theta, 0) A(0,0, \varphi),
\end{aligned}
$$


where $\phi \in[0, \pi), \theta \in[0, \pi / 2]$ and $\varphi \in[0, \pi)$ are determined uniquely by

$$
\cos (\theta)=|a|, \quad \arg (a)=\phi+\varphi, \quad \arg (b)=\pi+\phi-\varphi,
$$

if $a b \neq 0$. Now define a linear operator $\varrho_{d}(A) \in \mathrm{GL}\left(\mathcal{H}_{d}\right)$ by

$$
\varrho_{d}(A): f\left(z_{1}, z_{2}\right) \mapsto f\left(a z_{1}-b^{*} z_{2}, b z_{1}+a^{*} z_{2}\right) .
$$

It is straightforward to check that $\varrho_{d}$ is well defined.

Theorem 4.1. The mapping $\varrho_{d}: \mathrm{SU}(2) \rightarrow \mathrm{GL}\left(\mathcal{H}_{d}\right)$ is an irreducible unitary representation of degree $d$ of $\mathrm{SU}(2)$.

The proof of this result can be found in [47, Section III.2.3] (see also [26]). It turns out that the matrix representation of $\varrho_{d}(A)$ in the basis (4.1) (the so-called Wigner $d$-matrix) can be expressed by means of Jacobi polynomials. Indeed, introduce the function

$$
\mathbf{g}_{n}^{(\alpha, \beta)}(x)=\left(\frac{\Gamma(n+1) \Gamma(n+\alpha+\beta+1)}{\Gamma(n+\alpha+1) \Gamma(n+\beta+1)}\right)^{1 / 2}\left(\frac{1-x}{2}\right)^{\alpha / 2}\left(\frac{1+x}{2}\right)^{\beta / 2} P_{n}^{(\alpha, \beta)}(x) .
$$

Clearly, $\left\{\mathbf{g}_{n}^{(\alpha, \beta)}\right\}_{n \in \mathbb{N}_{0}}$ is an orthogonal system in $L^{2}(-1,1)$ and by (1.5)

$$
\int_{-1}^{1}\left|\mathbf{g}_{n}^{(\alpha, \beta)}(x)\right|^{2} d x=\frac{2}{2 n+\alpha+\beta+1} .
$$

Moreover, comparing (4.4) with (1.5), we get

$$
\mathbf{g}_{n}^{(\alpha, \beta)}(x)=\left(\frac{2}{2 n+\alpha+\beta+1}\right)^{1 / 2} \sqrt{w^{(\alpha, \beta)}(x)} \mathbf{p}_{n}^{(\alpha, \beta)}(x)
$$

Now we are ready to state the connection between $\varrho_{d}$ and Jacobi polynomials (see [47, Section III.3.9]).

Theorem 4.2. Let $A=A(\phi, \theta, \varphi) \in \mathrm{SU}(2), d \in \mathbb{N}$ and $\varrho_{d}$ be given by (4.3). Let also $l=(d-1) / 2$ and $k, j \in\{-l,-l+1, \ldots, l-1, l\}$. Then for all $k \geq 0$ and $|j| \leq k$

$$
\varrho_{d}(A)_{k, j}:=\left\langle\varrho_{d}(A) \psi_{j}^{d}, \psi_{k}^{d}\right\rangle_{\mathcal{H}_{d}}=\mathrm{e}^{-2 \mathrm{i}(k \phi+j \varphi)} \mathbf{g}_{l-k}^{(k-j, k+j)}(\cos (2 \theta)) .
$$

Since $\varrho_{d}(A)$ is a unitary matrix and $k \pm j \in \mathbb{N}_{0}$ in the formulation of Theorem 4.2, we immediately conclude that

$$
\left|\mathbf{g}_{n}^{(\alpha, \beta)}(x)\right| \leq 1
$$

for all $x \in[-1,1], \alpha, \beta \in \mathbb{N}_{0}$ and $n \in \mathbb{N}_{0}$. An analytic proof of a refined version of (4.8) can be found in [20] (see inequality (20) on p.234).

Lemma 4.3 (20]).

$$
\left|\mathbf{g}_{n}^{(\alpha, \beta)}(x)\right| \leq\left(\frac{(n+1)(n+\alpha+\beta+1)}{(n+\alpha+1)(n+\beta+1)}\right)^{1 / 4}
$$

for all $x \in[-1,1], \alpha, \beta \in \mathbb{N}_{0}$ and $n \in \mathbb{N}_{0}$.

Remark 4.4. Surprisingly enough we were not able to find the estimates (4.8) and (4.9) for noninteger values of $\alpha$ and $\beta$ in the literature. Numerically both seem to be true for noninteger values of $\alpha$ and $\beta$.

Let us also mention the following Bernstein-type inequality obtained recently by Haagerup and Schlichtkrull in [20]. 
Theorem 4.5 (20]). There is a constant $C<12$ such that

$$
\left|\left(1-x^{2}\right)^{1 / 4} \mathbf{g}_{n}^{(\alpha, \beta)}(x)\right| \leq \frac{C}{\sqrt[4]{2 n+\alpha+\beta+1}}
$$

for all $x \in[-1,1], \alpha, \beta \geq 0$ and $n \in \mathbb{N}_{0}$.

A few remarks are in order.

Remark 4.6. (i) The optimal value for the constant $C$ in (4.10) is not known.

(ii) The decay rate $n^{-1 / 4}$ in (4.10) is optimal as $\alpha$ and $\beta$ tend to infinity. However, for fixed $\alpha$ and $\beta$, the decay rate is $n^{-1 / 2}$ as $n \rightarrow \infty$ (see (1.12)).

(iii) It was observed in [20 that (4.10) implies the following interesting estimate for the matrix entries of $\varrho_{d}(A)$

$$
|\sin (2 \theta)|^{1 / 2}\left|\varrho_{d}(A(\phi, \theta, \varphi))_{j, k}\right| \leq C d^{-1 / 4},
$$

which provides the uniform decay $d^{-1 / 4}$ for the matrix coefficients, where $d$ is the dimension of the representation $\varrho_{d}$.

\section{UNIFORM WEIGHTED ESTIMATES FOR JACOBI POLYNOMIALS}

The main aim of this section is to prove the following inequality.

Theorem 5.1. The Bernstein-type estimate

$$
\left(\frac{1+x}{2}\right)^{\beta / 2}\left|P_{n}^{(\alpha, \beta)}(x)\right| \leq\left(\begin{array}{c}
n+\alpha \\
n
\end{array}\right), \quad x \in[-1,1],
$$

holds for all $n \in \mathbb{N}_{0}, \beta \geq 0$ and $\alpha \geq \beta-\lfloor\beta\rfloor$, where $\lfloor$.$\rfloor is the usual floor function.$

Equivalently, in terms of Meixner polynomials (3.4), we have

$$
c^{(n+x) / 2}\left|M_{n}(x ; \beta, c)\right| \leq 1, \quad x \geq n,
$$

where $0<c<1$ and $\beta \geq x-\lfloor x\rfloor+1$.

The proof is based on the product formula for biangle polynomials. More precisely, let

$$
\mathcal{B}=\left\{\left(x_{1}, x_{2}\right) \mid 0 \leq x_{2}^{2} \leq x_{1} \leq 1\right\}
$$

be the parabolic biangle. Following [22, 23], let $R_{n}^{(\alpha, \beta)}$ denote the Jacobi polynomials normalized by $R_{n}^{(\alpha, \beta)}(1)=1$, that is,

$$
R_{n}^{(\alpha, \beta)}(x)=\frac{P_{n}^{(\alpha, \beta)}(x)}{P_{n}^{(\alpha, \beta)}(1)} .
$$

For $\alpha, \beta>-1$ and $n, k \in \mathbb{N}_{0}$ such that $k \leq n$, define the parabolic biangle polynomials (see, e.g., [10, §2.6.1] and [24, §3.3], however, with a different notation)

$$
R_{n, k}^{\alpha, \beta}\left(x_{1}, x_{2}\right)=R_{n-k}^{(\alpha, \beta+k+1 / 2)}\left(2 x_{1}-1\right) \cdot x_{1}^{k / 2} R_{k}^{(\beta, \beta)}\left(x_{2} / \sqrt{x_{1}}\right), \quad\left(x_{1}, x_{2}\right) \in \mathcal{B} .
$$

Clearly, these functions are polynomials in $x_{1}$ and $x_{2}$ of degree $n$. Moreover, for fixed $\alpha$ and $\beta$ they are orthogonal with respect to the measure

$$
\left(1-x_{1}\right)^{\alpha}\left(x_{1}-x_{2}^{2}\right)^{\beta} d x_{1} d x_{2} .
$$

For certain values of $\alpha$ and $\beta$ the parabolic biangle polynomials have an interpretation as spherical functions for a Gelfand pair $(K, M)$, where $K$ is a compact group and $M$ is a closed subgroup. For these values of the parameters, the general theory 
of spherical functions on Gelfand pairs yields the existence of suitable product formulas and related hypergroup structures. The product formula in the general case was established in [27, Thm. 2.1]:

Theorem 5.2. Let $\alpha \geq \beta+1 / 2 \geq 0$. Let also $0 \leq\left|x_{2}\right| \leq x_{1} \leq 1$ and $0 \leq\left|y_{2}\right| \leq$ $y_{1} \leq 1$. If $\left(x_{1}, x_{2}\right),\left(y_{1}, y_{2}\right) \in \mathcal{B} \backslash\{(0,0)\}$, then the parabolic biangle polynomials satisfy the following hypergroup-type product formula:

$$
R_{n, k}^{\alpha, \beta}\left(x_{1}^{2}, x_{2}\right) \cdot R_{n, k}^{\alpha, \beta}\left(y_{1}^{2}, y_{2}\right)=\int_{I \times J^{3}} R_{n, k}^{\alpha, \beta}\left(E^{2}, E G\right) d \mu^{\alpha, \beta}\left(r_{1}, \psi_{1}, \psi_{2}, \psi_{3}\right),
$$

where $I=[0,1], J=[0, \pi]$,

$$
\begin{aligned}
D & =D(x, y ; r, \psi)=x y+\left(1-x^{2}\right)^{1 / 2}\left(1-y^{2}\right)^{1 / 2} r \cos \psi \\
E & =E\left(x_{1}, y_{1} ; r_{1}, \psi_{1}\right) \\
& =\left(x_{1}^{2} y_{1}^{2}+\left(1-x_{1}^{2}\right)\left(1-y_{1}^{2}\right) r_{1}^{2}+2 x_{1} y_{1}\left(1-x_{1}^{2}\right)^{1 / 2}\left(1-y_{1}^{2}\right)^{1 / 2} r_{1} \cos \psi_{1}\right)^{1 / 2}, \\
G & =D\left(\frac{D\left(x_{1}, y_{1} ; r_{1}, \psi_{1}\right)}{E\left(x_{1}, y_{1} ; r_{1}, \psi_{1}\right)}, D\left(\frac{x_{2}}{x_{1}}, \frac{y_{2}}{y_{1}} ; 1, \psi_{2}\right) ; 1, \psi_{3}\right),
\end{aligned}
$$

and

$$
\begin{aligned}
& d m^{\beta}(\psi)=\frac{\Gamma\left(\beta+\frac{3}{2}\right)}{\Gamma\left(\frac{1}{2}\right) \Gamma(\beta+1)}(\sin \psi)^{2 \beta+1} d \psi, \\
& d m^{-1}(\psi)=d\left[\frac{1}{2} \delta_{0}(\psi)+\frac{1}{2} \delta_{\pi}(\psi)\right], \\
& d m^{\alpha, \beta}(r, \psi)=\frac{2 \Gamma(\alpha+1)}{\Gamma(\alpha-\beta) \Gamma(\beta+1)}\left(1-r^{2}\right)^{\alpha-\beta-1} r^{2 \beta+1} d r d m^{\beta-\frac{1}{2}}(\psi), \\
& d m^{\alpha, \alpha}(r, \psi)=\frac{\Gamma(\alpha+1)}{\Gamma\left(\alpha+\frac{1}{2}\right) \Gamma\left(\frac{1}{2}\right)}(\sin \psi)^{2 \alpha} d\left(\delta_{1}\right)(r) d \psi, \\
& d \mu^{\alpha, \beta}\left(r, \psi_{1}, \psi_{2}, \psi_{3}\right)=d m^{\beta-\frac{1}{2}}\left(\psi_{3}\right) \cdot d m^{\beta-\frac{1}{2}}\left(\psi_{2}\right) \cdot d m^{\alpha, \beta+\frac{1}{2}}\left(r, \psi_{1}\right)
\end{aligned}
$$

are positive probability measures.

Before proving Theorem 5.1, we need the following simple fact.

Lemma 5.3. Let $X$ be a compact topological space and $X_{0}$ a dense subset of $X$. Suppose that $\phi \in C(X, \mathbb{R})$ such that for each $x, y \in X_{0}$ there is a (positive) probability Borel measure $\mu_{x, y}$ on $X$ with the property that

$$
\phi(x) \phi(y)=\int_{X} \phi(z) d \mu_{x, y}(z) .
$$

Then

$$
\max _{x \in X}|\phi(x)| \leq 1
$$

Proof. Let $M:=\max _{x \in X}|\phi(x)| \geq 0$. Then, from (5.7) we see

$$
\phi(x)^{2}=\phi(x) \phi(x)=\int_{X} \phi(z) d \mu_{x, x}(z) \leq M, \quad x \in X_{0} .
$$

Since $\phi$ is continuous and $X_{0}$ is dense in $X$, we infer $M^{2} \leq M$, that is, $M \leq 1$.

Remark 5.4. In the context of hypergroups Lemma 5.3 is well known as an inequality for bounded characters on a commutative hypergroup, see for instance the paper by Dunkl [9, Prop. 2.2(2)]. 
Proof of Theorem 5.1. Using the product formula and Lemma 5.3, we immediately conclude that

$$
\left|R_{n, k}^{\alpha, \beta}\left(x_{1}^{2}, x_{2}\right)\right| \leq 1, \quad\left(x_{1}, x_{2}\right) \in \mathcal{B},
$$

for all $k \leq n$ and $\alpha \geq \beta+1 / 2 \geq 0$. By (1.10) and (5.4) we know

$$
\left|R_{k}^{(\beta, \beta)}\left(x_{2} / \sqrt{x_{1}}\right)\right| \leq 1, \quad\left(x_{1}, x_{2}\right) \in \mathcal{B} \backslash\{(0,0)\}
$$

for all $k \in \mathbb{N}_{0}$ and $\beta \geq-1 / 2$, and hence we conclude (replacing $\beta+1 / 2$ by $\beta$ )

$$
\left(\frac{x+1}{2}\right)^{k / 2}\left|P_{n-k}^{(\alpha, \beta+k)}(x)\right| \leq\left(\begin{array}{c}
n-k+\alpha \\
n-k
\end{array}\right), \quad x \in[-1,1],
$$

for all $k \leq n$ and $\alpha \geq \beta \geq 0$. Since $n \in \mathbb{N}_{0}$ is arbitrary, we can replace $n-k$ by $n \in \mathbb{N}_{0}$. Moreover, choosing $\beta \in[0,1)$ and noting that $k \in \mathbb{N}_{0}$ is arbitrary, we finally end up with

$$
\left(\frac{x+1}{2}\right)^{\lfloor\beta\rfloor / 2}\left|P_{n}^{(\alpha, \beta)}(x)\right| \leq\left(\begin{array}{c}
n+\alpha \\
n
\end{array}\right), \quad x \in[-1,1],
$$

which holds for all $n \in \mathbb{N}_{0}$ and $\alpha \geq \beta-\lfloor\beta\rfloor$. Since $(x+1) / 2 \leq 1$ for all $x \in[-1,1]$, this completes the proof.

Remark 5.5. In fact, [27, Thm. 2.1] gives (5.6) also for the case $\left(x_{1}, x_{2}\right)=(0,0)$ or $\left(y_{1}, y_{2}\right)=(0,0)$, with a somewhat simpler measure on the right-hand side. Hence we might have worked with a version of Lemma 5.3 without the restriction to a dense subset.

We would like to finish this section with the following remark. We have two more proofs of Theorem 5.1 (see Appendix A), however, for a smaller set of parameters $\alpha$ and $\beta$. More precisely, using the addition formula for disk polynomials [23, one can prove (5.1) for all $\alpha \geq 0$ and $\beta \in \mathbb{N}_{0}$. The third proof is based on (4.8) and hence inherits the restriction $\alpha$ and $\beta \in \mathbb{N}_{0}$. It uses the Sonin-Pólya theorem 3 footnote to Theorem 7.31.1] and leads to the following result:

Theorem 5.6. Inequality (5.1) holds for all indices $\alpha, \beta$ for which (4.8) holds.

We firmly expect that (4.8) holds for all $x \in[-1,1]$ and $\alpha, \beta \geq 0$, which in particular would imply (5.1) for all $\alpha, \beta \geq 0$.

\section{Dispersion estimates for the eVOlution Group $\mathrm{e}^{-\mathrm{i} t H_{\alpha}}$}

It turns out that Theorem 3.1 (see also (3.15)) establishes a connection between Bernstein-type inequalities and dispersion estimates for the discrete Laguerre operators $H_{\alpha}$. In this section we shall present some $\ell^{1} \rightarrow \ell^{\infty}$ decay estimates for the evolution group $\mathrm{e}^{-\mathrm{i} t H_{\alpha}}$ based on Bernstein-type inequalities from the previous sections.

First, notice that (3.15) can be rewritten in terms of the function $\mathbf{g}_{n}^{(\alpha, \beta)}$ introduced in (4.4):

$$
\left|\mathrm{e}^{-\mathrm{i} t H_{\alpha}}(n, m)\right|=\frac{1}{\sqrt{1+t^{2}}}\left|\mathbf{g}_{n}^{(\alpha, m-n)}\left(\frac{t^{2}-1}{t^{2}+1}\right)\right|, \quad m \geq n .
$$

Hence the estimate (4.8) immediately implies

\footnotetext{
${ }^{3}$ In the literature Sonin is also written as Sonine.
} 
Theorem 6.1. Let $\alpha \in \mathbb{N}_{0}$. Then the following estimate

$$
\left\|\mathrm{e}^{-\mathrm{i} t H_{\alpha}}\right\|_{\ell^{1} \rightarrow \ell^{\infty}} \leq \frac{1}{\sqrt{1+t^{2}}}, \quad t \in \mathbb{R}
$$

holds. Moreover, in the case $\alpha=0$, the inequality can be replaced by equality.

Proof. To prove the last claim it suffices to note that

$$
\left\|\mathrm{e}^{-\mathrm{i} t H_{0}}\right\|_{\ell^{1} \rightarrow \ell^{\infty}} \geq\left|\mathrm{e}^{-\mathrm{i} t H_{0}}(0,0)\right|=\frac{1}{\sqrt{1+t^{2}}}
$$

for all $t \in \mathbb{R}$.

Remark 6.2. (i) The case $\alpha=0$ was proven in 28]. Using a different approach, a weaker estimate in the case $\alpha=0$ was obtained in 34 .

(ii) Using Lemma 4.3 (see also (4.8)), we get the somewhat stronger estimate

$$
\left|\mathrm{e}^{-\mathrm{i} t H_{\alpha}}(n, m)\right| \leq \frac{1}{\sqrt{1+t^{2}}}\left(\frac{(n+1)(m+\alpha+1)}{(m+1)(n+\alpha+1)}\right)^{1 / 4}
$$

which holds for all $m \geq n, \alpha \in \mathbb{N}_{0}$ and $t \in \mathbb{R}$.

Conjecture 6.1. We conjecture that (4.8) as well as Lemma 4.3 hold true for all $\alpha, \beta \geq 0$ and consequently Theorem 6.1 holds for all $\alpha \geq 0$.

Applying the Haagerup-Schlichtkrull inequality (4.10) to (6.1) we obtain another estimate, which holds for all $\alpha \geq 0$ :

Theorem 6.3. Let $\alpha \geq 0$. There is a positive constant $C<6 \sqrt{2}$ such that the following inequality

$$
\left|\mathrm{e}^{-\mathrm{i} t H_{\alpha}}(n, m)\right| \leq \frac{C|t|^{-1 / 2}}{\sqrt[4]{n+m+\alpha+1}},
$$

holds for all $n, m \in \mathbb{N}_{0}$ and $t \neq 0$.

Remark 6.4. (i) The estimate in Theorem 6.3 provides only a $t^{-1 / 2}$ decay, however, it gives an $(n+m)^{-1 / 4}$ decay of the matrix coefficients.

(ii) Let us also mention that the Erdélyi-Magnus-Nevai conjecture (1.14) would imply the following estimate

$$
\left|\mathrm{e}^{-\mathrm{i} t H_{\alpha}}(n, m)\right| \leq \frac{C}{\sqrt{|t|}} \frac{(\alpha+|m-n|)^{1 / 4}}{(n+m+\alpha+1)^{1 / 2}}, \quad t \neq 0 .
$$

The latter shows that on diagonals, i.e., when $m-n=$ const, the decay of the matrix elements is $n^{-1 / 2}$ as $n \rightarrow \infty$. However, it does not improve (6.3) when $m-n$ tends to infinity.

The estimates (6.2) and (6.3) provide a non-integrable decay as $t \rightarrow \infty$. However, in order to establish stability for soliton type solutions to nonlinear equations it is desirable to have an integrable decay in $t$. As we mentioned in Section 3, we expect a decay of order $O\left(|t|^{-(1+\alpha)}\right)$, however, in weighted spaces. To this end let $\sigma=\{\sigma(n)\}_{n \geq 0}$ be a positive sequence. Consider the weighted $\ell^{p}$ spaces equipped with the norm

$$
\|u\|_{\ell^{p}(\sigma)}= \begin{cases}\left(\sum_{n \in \mathbb{N}_{0}} \sigma(n)|u(n)|^{p}\right)^{1 / p}, & p \in[1, \infty), \\ \sup _{n \in \mathbb{N}_{0}} \sigma(n)|u(n)|, & p=\infty .\end{cases}
$$


Of course, the case $\sigma \equiv 1$ corresponds to the usual $\ell^{p}(\sigma)=\ell^{p}$ spaces without weight. Specifically we will work with the weights $\sigma_{\alpha}(n)$, given in (2.5), and consider the weighted spaces $\ell^{1}\left(\sigma_{\alpha}\right)$ and $\ell^{\infty}\left(\sigma_{\alpha}^{-1}\right)$. Notice that

$$
\sigma_{\alpha}(n)=\frac{n^{\alpha / 2}}{\sqrt{\Gamma(\alpha+1)}}(1+o(1)), \quad n \rightarrow \infty .
$$

Theorem 6.5. The following equality

$$
\left\|\mathrm{e}^{-\mathrm{i} t H_{\alpha}}\right\|_{\ell^{1}\left(\sigma_{\alpha}\right) \rightarrow \ell^{\infty}\left(\sigma_{\alpha}^{-1}\right)}=\left(\frac{1}{1+t^{2}}\right)^{\frac{1+\alpha}{2}}, \quad t \in \mathbb{R},
$$

holds for all $\alpha \geq 0$.

Proof. First of all, noting that $\mathrm{e}^{-\mathrm{i} t H_{\alpha}}(0,0)=(1+\mathrm{i} t)^{-1-\alpha}$ (see Corollary 3.5 (i)), we get

$$
\left\|\mathrm{e}^{-\mathrm{i} t H_{\alpha}}\right\|_{\ell^{1}\left(\sigma_{\alpha}\right) \rightarrow \ell^{\infty}\left(\sigma_{\alpha}^{-1}\right)} \geq\left(\frac{1}{1+t^{2}}\right)^{\frac{1+\alpha}{2}}, \quad t \in \mathbb{R} .
$$

The converse inequality

$$
\frac{\left|\mathrm{e}^{-\mathrm{i} t H_{\alpha}}(n, m)\right|}{\sigma_{\alpha}(n) \sigma_{\alpha}(m)} \leq\left(\frac{1}{1+t^{2}}\right)^{\frac{1+\alpha}{2}}, \quad t \in \mathbb{R}, n, m \in \mathbb{N}_{0},
$$

follows from the Bernstein-type estimate (5.1). Indeed, by (3.2), it suffices to consider the case $n \leq m$. Using (3.15) and making the change of variables (3.14), we get

$$
\left(1+t^{2}\right)^{\frac{1+\alpha}{2}} \frac{\mathrm{e}^{-\mathrm{i} t H_{\alpha}}(n, m) \mid}{\sigma_{\alpha}(n) \sigma_{\alpha}(m)}=\left(\begin{array}{c}
n+\alpha \\
n
\end{array}\right)^{-1}\left(\frac{1+x}{2}\right)^{\frac{m-n}{2}}\left|P_{n}^{(\alpha, m-n)}(x)\right| .
$$

However, by (5.1), the right-hand side is less than 1, which completes the proof.

Remark 6.6. An inspection of $\mathrm{e}^{-\mathrm{i} t H_{\alpha}}(n, m)$ with $n=m=1$ (see the proof of Corollary [3.9) shows that (5.1) is no longer true for $\alpha<0$. However, we expect that the following estimate

$$
\left\|\mathrm{e}^{-\mathrm{i} t H_{\alpha}}\right\|_{\ell^{1} \rightarrow \ell \infty}=\mathcal{O}\left(|t|^{-(1+\alpha)}\right), \quad t \rightarrow \infty,
$$

holds true for all $\alpha \in(-1,0)$.

\section{Conclusions}

7.1. A hunt for Bernstein-type inequalities. The main aim of this paper was to prove dispersive decay for the evolution group $\mathrm{e}^{-\mathrm{i} t H_{\alpha}}$. It turned out that this problem is closely related to Bernstein-type inequalities for (1.11) and, in particular, has led us to new Bernstein-type inequalities (5.1) and (3.13). In fact, the search for an optimal decay in $t$ or in $m$ and $n$ for the kernel $\mathrm{e}^{-\mathrm{i} t H_{\alpha}}(n, m)$ leads to a wider class of Bernstein-type inequalities. More precisely, recall the change of variables (3.14) and let $\eta \in[0,1+\alpha], \nu \geq 0$ be fixed. Then (6.8), after substitution of (2.5), can be rewritten as

$$
\begin{aligned}
& \left(1+t^{2}\right)^{\frac{\eta}{2}}\left|\mathrm{e}^{-\mathrm{i} t H_{\alpha}}(n, m)\right|\left(\frac{t^{2}}{1+t^{2}}\right)^{\frac{\nu}{2}}= \\
& \frac{\sigma_{\alpha}(m)}{\sigma_{\alpha}(n)}\left(\frac{1+x}{2}\right)^{\frac{m-n+\nu}{2}}\left(\frac{1-x}{2}\right)^{\frac{1+\alpha-\eta}{2}}\left|P_{n}^{(\alpha, m-n)}(x)\right|,
\end{aligned}
$$


for all $n \leq m$. Let $\sigma=\{\sigma(n)\}_{n \geq 0}$ be a positive weight. Noting that

$$
\left\|\mathrm{e}^{-\mathrm{i} t H_{\alpha}}\right\|_{\ell^{1}(\sigma) \rightarrow \ell^{\infty}\left(\sigma^{-1}\right)}=\sup _{n, m \in \mathbb{N}_{0}} \sigma(n)^{-1}\left|\mathrm{e}^{-\mathrm{i} t H_{\alpha}}(n, m)\right| \sigma(m)^{-1},
$$

we conclude that the dispersive decay estimate

$$
\left\|\mathrm{e}^{-\mathrm{i} t H_{\alpha}}\right\|_{\ell^{1}(\sigma) \rightarrow \ell^{\infty}\left(\sigma^{-1}\right)} \leq C\left(1+t^{2}\right)^{-\eta / 2}, \quad t \in \mathbb{R},
$$

would follow from the Bernstein-type bound

$$
\left(\frac{1-x}{2}\right)^{\frac{1+\alpha-\eta}{2}}\left(\frac{1+x}{2}\right)^{\frac{m-n+\nu}{2}}\left|P_{n}^{(\alpha, m-n)}(x)\right| \leq C \sigma(n) \sigma(m) \sqrt{\frac{(\alpha+1)_{n} m !}{(\alpha+1)_{m} n !}}
$$

for all $n \leq m$ and $x \in(-1,1)$. Clearly, the latter is a uniform weighted estimate for (1.11) with $a=\frac{1+\alpha-\eta}{2}$ and $b=\frac{\beta+\nu}{2}$. In this respect let us mention that our Theorem 5.1 gives rise to $\eta=1+\alpha$ and $\nu=0$; the estimates (4.8)-(4.9) correspond to the case $\eta=1$ and $\nu=0$; the Erdelyi-Magnus-Nevai conjecture (1.14) and the Haagerup-Schlichtkrull inequality (4.10) correspond to $\eta=\nu=1 / 2$.

7.2. 1-D spherical Schrödinger operators. Let us finish this paper by comparing our results with the recent study of dispersive estimates for the one-dimensional spherical Schrödinger operators

$$
\mathrm{H}_{l}=-\frac{d^{2}}{d x^{2}}+\frac{l(l+1)}{x^{2}}, \quad l \geq-\frac{1}{2}
$$

acting in $L^{2}\left(\mathbb{R}_{+}\right)\left(\mathrm{H}_{l}\right.$ denotes the Friedrichs extension if $\left.l \in(-1 / 2,1 / 2)\right)$. In the free case $l=0$, one has

$$
\left\|\mathrm{e}^{-\mathrm{i} t \mathrm{H}_{0}}\right\|_{L^{1}\left(\mathbb{R}_{+}\right) \rightarrow L^{\infty}\left(\mathbb{R}_{+}\right)}=\mathcal{O}\left(|t|^{-1 / 2}\right), \quad t \rightarrow \infty .
$$

It was shown in [30] (see also [29]) that $\left\|\mathrm{e}^{-\mathrm{i} t \mathrm{H}_{l}}\right\|_{L^{1} \rightarrow L^{\infty}}=\mathcal{O}\left(|t|^{-1 / 2}\right)$ as $t \rightarrow \infty$ for all $l \geq-1 / 2$. On the other hand, considering weighted $L^{1} \rightarrow L^{\infty}$ estimates, one can improve the decay in $t$ for positive $l>0$ [29, 30]:

$$
\left\|\mathrm{e}^{-\mathrm{i} t \mathrm{H}_{l}}\right\|_{L^{1}\left(\mathbb{R}_{+} ; x^{l}\right) \rightarrow L^{\infty}\left(\mathbb{R}_{+} ; x^{-l}\right)}=\mathcal{O}\left(|t|^{-l-1 / 2}\right), \quad t \rightarrow \infty .
$$

Since $\alpha$ in (1.17) can be seen as a measure of the delocalization of the field configuration and it is related to the planar angular momentum [2, our dispersive decay estimates (6.2) and (6.6) can be viewed as analogues of the above mentioned results for spherical Schrödinger operators from [29, 30].

\section{Appendix A. Two alternate proof For the main theorem}

A.1. The addition formula for disk polynomials. Following [22, 23], let $R_{n}^{(\alpha, \beta)}$ denote the Jacobi polynomials normalized as in (5.4). Consider the disk polynomials (see, e.g., [10, §2.6.3] and [24, §3.3], however, with a different notation)

$$
R_{m, n}^{(\alpha)}\left(r \mathrm{e}^{\mathrm{i} \phi}\right)=r^{|m-n|} \mathrm{e}^{\mathrm{i}(m-n) \phi} R_{\min (m, n)}^{(\alpha,|m-n|)}\left(2 r^{2}-1\right) .
$$

For $\alpha=q-2$ with an integer $q \geq 2$ and under a suitable choice of coordinates on the unit sphere $\mathbb{S}^{2 q}$ in $\mathbb{C}^{q}$, these functions are zonal surface harmonics of type $(m, n)$ as introduced by Ikeda 21. This interpretation of disk polynomials was the key to the following addition formula established in [22, 23]. 
Theorem A.1 (23]). Let $\alpha>0$. The following addition formula holds:

$$
\begin{aligned}
& R_{m, n}^{(\alpha)}\left(\cos \left(\theta_{1}\right) \mathrm{e}^{\mathrm{i} \phi_{1}} \cos \left(\theta_{2}\right) \mathrm{e}^{\mathrm{i} \phi_{2}}+\sin \left(\theta_{1}\right) \sin \left(\theta_{2}\right) r \mathrm{e}^{\mathrm{i} \psi}\right) \\
& =\sum_{k=0}^{m} \sum_{l=0}^{n} c_{m, n, k, l}^{(\alpha)}\left(\sin \left(\theta_{1}\right)\right)^{k+l} R_{m-k, n-l}^{(\alpha+k+l)}\left(\cos \left(\theta_{1}\right) \mathrm{e}^{\mathrm{i} \phi_{1}}\right) \\
& \quad \times\left(\sin \left(\theta_{2}\right)\right)^{k+l} R_{m-k, n-l}^{(\alpha+k+l)}\left(\cos \left(\theta_{2}\right) \mathrm{e}^{\mathrm{i} \phi_{2}}\right) R_{k, l}^{(\alpha-1)}\left(r \mathrm{e}^{\mathrm{i} \psi}\right),
\end{aligned}
$$

where

$$
c_{m, n, k, l}^{(\alpha)}=\frac{\alpha}{\alpha+k+l}\left(\begin{array}{c}
m \\
k
\end{array}\right)\left(\begin{array}{c}
n \\
l
\end{array}\right) \frac{(\alpha+n+1)_{k}(\alpha+m+1)_{l}}{(\alpha+l)_{k}(\alpha+k)_{l}} .
$$

The addition formula (A.2) leads to (5.1) for integer $\beta$.

Proof of Theorem [5.1: The case $\alpha>0, \beta \in \mathbb{N}_{0}$. Setting $\theta_{1}=\theta_{2}=\theta \in[0, \pi], \phi_{1}=$ $\phi_{2}=\psi=0, r=1$ in (A.2) and assuming $n \leq m$, we end up with

$$
1=R_{m, n}^{(\alpha)}(1)=\sum_{k=0}^{m} \sum_{l=0}^{n} c_{m, n, k, l}^{(\alpha)}(\sin (\theta))^{2(k+l)} R_{m-k, n-l}^{(\alpha+k+l)}(\cos (\theta))^{2} .
$$

In particular, since all summands are nonnegative and using the normalization, we easily get the following estimate (notice that $c_{m, n, 0,0}^{(\alpha)}=1$ )

$$
R_{m, n}^{(\alpha)}(\cos (\theta))^{2} \leq 1, \quad \theta \in[0, \pi],
$$

which proves the claim after a simple change of variables. Then the case $\alpha=0$, $\beta \in \mathbb{N}_{0}$ follows by continuity.

Remark A.2. A different proof of (A.5) can be found in [10, Proposition 2.6.7(i)].

A.2. The Sonin-Polyá theorem. Here we provide a direct proof for Theorem 5.1 using the Sonin-Pólya theorem and the inequality (4.8), which gives some further insight into the behavior of the left-hand side of (5.1).

We divide the proof in three steps. First, let us establish an explicit neighborhood of $x=1$ where (5.1) holds. For this we recall the Sonin-Pólya theorem 44, footnote to Theorem 7.31.1], which associates with a solution $y$ of a differential equation

$$
\left(p y^{\prime}\right)^{\prime}+q y=0
$$

a Sonin function

$$
S(x):=y(x)^{2}+\frac{p(x)}{q(x)} y^{\prime}(x)^{2}
$$

and then we observe that

$$
S^{\prime}(x)=-(p q)^{\prime}(x)\left(\frac{y^{\prime}(x)}{q(x)}\right)^{2},
$$

by which successive relative maxima of $y^{2}$ form an increasing or decreasing sequence according as $p q$ decreases or increases on the corresponding interval.

Lemma A.3. Let $\alpha, \beta \geq 0$ and $n \in \mathbb{N}_{0}$. Put $\lambda_{n}=n(n+\alpha+\beta+1)$. There are points

$$
-1 \leq x_{0} \leq x_{1} \leq 1
$$


given explicitly by

$$
x_{0}=-1+\frac{2 \beta^{2}}{\beta^{2}+2 \beta(1+\alpha)+4 \lambda_{n}}, \quad x_{1}=1-\frac{2(1+2 \alpha)\left(\beta+\beta \alpha+2 \lambda_{n}\right)}{(1+\alpha)\left(\beta^{2}+2 \beta(1+\alpha)+4 \lambda_{n}\right)},
$$

such that the relative maxima of

$$
\left(\frac{1+x}{2}\right)^{\beta}\left|P_{n}^{(\alpha, \beta)}(x)\right|^{2}
$$

are increasing on $\left(x_{1}, 1\right]$ and decreasing on $\left(x_{0}, x_{1}\right)$. Moreover, inequality (5.1) holds on $\left[x_{1}, 1\right]$ and there are no relative maxima on $\left[-1, x_{0}\right)$.

Proof. Abbreviate

$$
y(x):=\left(\frac{1+x}{2}\right)^{\beta / 2} P_{n}^{(\alpha, \beta)}(x) .
$$

Then rewriting of the differential equation [44, (4.2.1)] for Jacobi polynomials shows that $y$ satisfies (A.6) with

$$
p(x)=(1-x)^{\alpha+1}(1+x) \quad \text { and } \quad 4 p(x) q(x)=f(x)(1-x)^{2 \alpha+1},
$$

where

$$
f(x)=-\beta^{2}+2 \beta(1+\alpha)+4 \lambda_{n}+x\left(\beta^{2}+2 \beta(1+\alpha)+4 \lambda_{n}\right) .
$$

The corresponding Sonin function $S$ given by (A.7) then has a singularity at the zero $x_{0}$ of $f$ such that $S(x) \rightarrow-\infty$ or $+\infty$ according as $x \uparrow x_{0}$ or $x \downarrow x_{0}$. Then a calculation shows that

$$
4(1-x)^{-2 \alpha}(p q)^{\prime}(x)=\left(\beta^{2}+2 \beta(1+\alpha)+4 \lambda_{n}\right)(1-x)-(2 \alpha+1) f(x),
$$

which has a zero at $x_{1}$. The inequalities (A.8) are easily checked. Now we see that $S(x)$ decreases from 0 to $-\infty$ on $\left[-1, x_{0}\right)$, decreases from $\infty$ to $S\left(x_{1}\right)$ on $\left(x_{0}, x_{1}\right]$ and increases from $S\left(x_{1}\right)$ to $S(1)=P_{n}^{(\alpha, \beta)}(1)^{2}$ on $\left[x_{1}, 1\right]$. In particular, $S(x)<0$ on $\left(-1, x_{0}\right)$ and hence there cannot be any maxima of $y(x)^{2}$ in this interval.

Now let us find an explicit neighborhood of $x=-1$ where (5.1) holds.

Lemma A.4. Inequality (5.1) holds on the interval

$$
\left[-1, x_{2}\right), \quad x_{2}=1-2\left(\left(\begin{array}{c}
n+\alpha \\
n
\end{array}\right)\left(\begin{array}{c}
n+\alpha+\beta \\
n+\beta
\end{array}\right)\right)^{-1 / \alpha}
$$

for all indices $\beta, \alpha$ for which (4.8) holds.

Proof. This follows upon inserting (4.8) into the desired inequality (5.1) and solving for $x$.

We need the following technical lemma, which allows to estimate $x_{2}$.

Lemma A.5. The following inequality for binomial coefficients holds for all $x \geq 0$ :

$$
\left(\begin{array}{c}
x+y \\
x
\end{array}\right) \geq \begin{cases}(x+y)^{y}, & 0 \leq y \leq 1 \\
\left(\frac{x+y}{y}\right)^{y} & 1 \leq y\end{cases}
$$

Remark A.6. The case $0 \leq y \leq 1$ follows from an inequality due to Wendel 49 , eqn. (7)]. The case $1 \leq y$ can be found in, e.g., [41, eqn. (41)] (as written there after the formula: the reverse inequality (41) holds if $a>1$ ).

Finally we note: 
Lemma A.7. Suppose $\alpha \geq 1$ and $n \geq 1$ or $\alpha \geq 0$ and $n \geq 2$. Then

$$
x_{1} \leq x_{2} .
$$

Proof. First of all note that $x_{1} \leq x_{2}$ is equivalent to

$$
\left(\left(\begin{array}{c}
n+\alpha \\
n
\end{array}\right)\left(\begin{array}{c}
n+\alpha+\beta \\
n+\beta
\end{array}\right)\right)^{-1 / \alpha} \leq \frac{(1+2 \alpha)\left(\beta(1+\alpha)+2 \lambda_{n}\right)}{(1+\alpha)\left(\beta^{2}+2 \beta(1+\alpha)+4 \lambda_{n}\right)} .
$$

Now inequality (A.9) for $\alpha \geq 1$ implies that $x_{1} \leq x_{2}$ will hold if

$$
\frac{\alpha}{n+\alpha} \frac{\alpha}{n+\alpha+\beta} \leq \frac{(1+2 \alpha)\left(\beta(1+\alpha)+2 \lambda_{n}\right)}{(1+\alpha)\left(\beta^{2}+2 \beta(1+\alpha)+4 \lambda_{n}\right)} .
$$

However, it is easy to check that a stronger inequality (note that $1+\alpha \leq n+\alpha$ for $n \geq 1$ )

$$
\alpha\left(\beta^{2}+2 \beta(1+\alpha)+4 \lambda_{n}\right) \leq 2\left(\beta(1+\alpha)+2 \lambda_{n}\right)(n+\alpha+\beta)
$$

holds true for $n \geq 1$ and $\beta, \alpha \geq 0$. The case $0 \leq \alpha \leq 1$ is similar.

Combining Lemmas A.3, A.4 and A.7, we arrive at Theorem 5.6.

Proof of Theorem 5.6. Since the cases $n=0,1$ can be checked directly (see Corollary 3.9), combining Lemmas A.3, A.4 and A.7, we conclude that (5.1) holds for all $\alpha, \beta \geq 0$ for which (4.8) holds and hence in particular for $\alpha, \beta \in \mathbb{N}_{0}$.

Acknowledgments. We are indebted to Alexander Aptekarev, Christian Krattenthaler, Margit Rösler, Walter Van Assche and Michael Voit for discussions on these topics. We also thank the referees for the careful reading of our manuscript.

\section{REFERENCES}

[1] C. Acatrinei, Noncommutative radial waves, J. Phys. A: Math. Theor. 41, 215401 (2008).

[2] C. Acatrinei, Discrete nonlocal waves, JHEP 02, 057 (2013).

[3] N. I. Akhiezer, The Classical Moment Problem and Some Related Questions in Analysis, Oliver and Boyd Ltd., Edinburgh, London, 1965.

[4] V. A. Antonov and K. V. Holševnikov, An estimate of the remainder in the expansion of the generating function for the Legendre polynomials (Generalization and improvement of Bernstein's inequality), Vestnik Leningrad Univ. Math. 13, 163-166 (1981).

[5] D. Basu and K. B. Wolf, The unitary irreducible representations of $\operatorname{SL}(2, \mathbb{R})$ in all subgroup reductions, J. Math. Phys. 23, 189-205 (1982).

[6] N. Burq, S. Dyatlov, R. Ward, and M. Zworski, Weighted eigenfunction estimates with applications to compressed sensing, SIAM J. Math. Anal. 44, no. 5, 3481-3501 (2012).

[7] T. Chen, J. Fröhlich, and J. Walcher, The decay of unstable noncommutative solitons, Commun. Math. Phys. 237, 243-269 (2003).

[8] Y. Chow, L. Gatteschi, and R. Wong, A Bernstein-type inequality for the Jacobi polynomial, Proc. Amer. Math. Soc. 121, 703-709 (1994).

[9] C. F. Dunkl, The measure algebra of a locally compact hypergroup, Trans. Amer. Math. Soc. 179, 331-348 (1973).

[10] C. F. Dunkl and Y. Xu, Orthogonal Polynomials of Several Variables, 2nd. ed., Encyclopedia Math. Appl. 155, Cambridge Univ. Press, 2014.

[11] A. Erdélyi et al., Higher Transcendental Functions, Vol. 1, McGraw-Hill, New York, 1953.

[12] A. Erdélyi et al., Tables of Integral Transforms, Vol. 1, McGraw-Hill, New York, 1954.

[13] T. Erdélyi, A. P. Magnus, and P. Nevai, Generalized Jacobi weights, Christoffel functions, and Jacobi polynomials, SIAM J. Math. Anal. 25, 602-614 (1994).

[14] K.-J. Förster, Inequalities for ultraspherical polynomials and application for quadratures, J. Comp. Appl. Math. 49, 59-70 (1993).

[15] W. Gautschi, How sharp is Bernstein's inequality for Jacobi polynomials?, Electron. Trans. Numer. Anal. 36, 1-8 (2009/2010). 
[16] R. Gopakumar, S. Minwalla, and A. Strominger, Noncommutative solitons, JHEP 05, 020 (2000).

[17] U. Haagerup and J. Kraus, Approximation properties for group $C^{*}$-algebras and group von Neumann algebras, Trans. Amer. Math. Soc. 344, 667-699 (1994).

[18] U. Haagerup and T. de Laat, Simple Lie groups without the approximation property, Duke Math. J. 162, 925-964 (2013).

[19] U. Haagerup and T. de Laat, Simple Lie groups without the approximation property II, Trans. Amer. Math. Soc. 368, 3777-3809 (2016).

[20] U. Haagerup and H. Schlichtkrull, Inequalities for Jacobi polynomials, Ramanujan J. 33, $227-246$ (2014).

[21] M. Ikeda, On spherical functions for the unitary group. I, II, III, Mem. Fac. Engrg. Hiroshima Univ. 3, 17-29, 31-53, 55-75 (1967).

[22] T. H. Koornwinder, The addition formula for Jacobi polynomials II. The Laplace type integral and the product formula, Report TW 133/72. Mathematisch Centrum, Amsterdam, 29 pp. (1972) https://staff.fnwi.uva.nl/t.h.koornwinder/art/index.html\#1972

[23] T. H. Koornwinder, The addition formula for Jacobi polynomials III. Completion of the proof, Report TW 135/72. Mathematisch Centrum, Amsterdam, 11 pp. (1972) https://staff.fnwi.uva.nl/t.h.koornwinder/art/index.html\#1972

[24] T. H. Koornwinder, Two-variable analogues of the classical orthogonal polynomials, in: R. A. Askey (ed.) "Theory and Applications of Special Functions", pp. 434-495, Acad. Press, New York, San Francisco, London, 1975.

[25] T. H. Koornwinder, Group theoretic interpretations of Askey's scheme of hypergeometric orthogonal polynomials, in: M. Alfaro et. al. (eds.) "Orthogonal polynomials and their applications", pp. 46-72, Lecture Notes Math. 1329, Springer-Verlag, Berlin, 1988.

[26] T. H. Koornwinder, Representations of $\mathrm{SU}(2)$ and Jacobi polynomials, arXiv:1606.08189

[27] T. H. Koornwinder and A. L. Schwartz, Product formulas and associated hypergroups for orthogonal polynomials on the simplex and on a parabolic biangle, Constr. Approx. 11, 537567 (1997).

[28] A. Kostenko and G. Teschl, Dispersion estimates for the discrete Laguerre operator, Lett. Math. Phys. 106, no. 4, 545-555 (2016).

[29] A. Kostenko, G. Teschl and J. H. Toloza, Dispersion estimates for spherical Schrödinger equations, Ann. Henri Poincaré 17, no. 11, 3147-3176 (2016).

[30] H. Kovařík and F. Truc, Schrödinger operators on a half-line with inverse square potentials, Math. Model. Nat. Phenom. 9, no. 5, 170-176 (2014).

[31] I. Krasikov, An upper bound on Jacobi polynomials, J. Approx. Theory 149, 116-130 (2007)

[32] I. Krasikov, On Erdélyi-Magnus-Nevai conjecture for Jacobi polynomials, Constr. Approx. 28, 113-125 (2008)

[33] A. J. Krueger and A. Soffer, Structure of noncommutative solitons: existence and spectral theory, Lett. Math. Phys. 105, 1377-1398 (2015).

[34] A. J. Krueger and A. Soffer, Dynamics of noncommutative solitons I: Spectral theory and dispersive estimates, Ann. Henri Poincaré 17, 1181-1208 (2016).

[35] A. J. Krueger and A. Soffer, Dynamics of noncommutative solitons II: Spectral theory, dispersive estimates and stability, arXiv:1411.5859

[36] V. Lafforgue and M. de la Salle, Non commutative $L^{p}$ spaces without the completely bounded approximation property, Duke Math. J. 160, 71-116 (2011).

[37] G. Lohöfer, Inequalities for Legendre functions and Gegenbauer functions, J. Approx. Theory 64, 226-234 (1991).

[38] L. Lorch, Alternative proof of a sharpened form of Bernstein's inequality for Legendre polynomials, Appl. Anal. 14, no. 3, 237-240 (1982/83).

[39] L. Lorch, Inequalities for ultraspherical polynomials and the gamma function, J. Approx. Theory 40, no. 2, 115-120 (1984).

[40] F. W. J. Olver et al., NIST Handbook of Mathematical Functions, Cambridge University Press, Cambridge, 2010; http://dlmf.nist.gov .

[41] F. Qi, D.-W. Niu, J. Cao and S.-X. Chen, Four logarithmically completely monotonic functions involving gamma function, J. Korean Math. Soc. 45, 559-573 (2008).

[42] H. Rauhut and R. Ward, Sparse recovery for spherical harmonic expansions, in: Proceedings of the 9th International Conference on Sampling Theory and Applications (SampTA 2011), Singapore, 2011 (arXiv:1102.4097). 
[43] P. J. Sally, Jr., Analytic continuation of the irreducible unitary representations of the universal covering group of $\mathrm{SL}(2, \mathbb{R})$, Memoirs Amer. Math. Soc. 69, Amer. Math. Soc., 1967.

[44] G. Szegö, Orthogonal Polynomials, Amer. Math. Soc., Providence, RI, Fourth edition, 1975.

[45] G. Teschl, Jacobi Operators and Completely Integrable Nonlinear Lattices, Math. Surv. and Mon. 72, Amer. Math. Soc., Rhode Island, 2000.

[46] G. Teschl, Mathematical Methods in Quantum Mechanics; With Applications to Schrödinger Operators, 2nd ed., Amer. Math. Soc., Rhode Island, 2014.

[47] N. Ja. Vilenkin, Special Functions and the Theory of Group Representations, Amer. Math. Soc., Providence, 1968.

[48] N. Ja. Vilenkin and A. U. Klimyk, Representation of Lie Groups and Special Functions, Vol. 1, Kluwer, Dordrecht, 1991.

[49] J. G. Wendel, Note on the gamma function, Amer. Math. Monthly 55, 563-564 (1948).

Korteweg-De Vries Institute for Mathematics, University of Amsterdam, 1090 GE Amsterdam, Netherlands

E-mail address: T.H.Koornwinder@uva.nl

$U R L:$ https://staff.fnwi.uva.nl/t.h.koornwinder/

Faculty of Mathematics and Physics, University of Ljubljana, Jadranska ul. 19, 1000 Ljubljana, Slovenia, and Faculty of Mathematics, University of Vienna, OskarMorgenstern-Platz 1, 1090 Vienna, Austria

E-mail address: Aleksey.Kostenko@fmf.uni-lj.si; Oleksiy.Kostenko@univie.ac.at

$U R L:$ http://www.mat.univie.ac.at/ kostenko/

Faculty of Mathematics, University of Vienna, Oskar-Morgenstern-Platz 1, 1090 Wien, Austria, and International Erwin Schrödinger Institute for Mathematical Physics, Boltzmanngasse 9, 1090 Wien, Austria

E-mail address: Gerald.Teschl@univie.ac.at

$U R L:$ http://www.mat.univie.ac.at/ gerald/ 